\title{
Case study on diagnosis and identify the degree of bottom hole liquid accumulation in double-branch horizontal wells in PCOC
}

\author{
Tingwei Yao ${ }^{1}$, Yang Zhang ${ }^{1}$, Minhao Guo ${ }^{2}$, Zhilin Tuo ${ }^{1}$, Haiyang Wang, ${ }^{1, *}$, and Desheng Zhou ${ }^{1,3}$ \\ ${ }^{1}$ College of Petroleum Engineering, Xi'an Shiyou University, Xi'an, 710065, China \\ ${ }^{2}$ The College of Life Sciences, Northwest University, Xi'an, 710069, China \\ ${ }^{3}$ Engineering Research Center of Development and Management for Low to Extra-Low Permeability Oil \& Gas Reservoirs in West \\ China, Ministry of Education, Xi'an Shiyou University, Xi'an, 710065, Shaanxi, China
}

\begin{abstract}
In the process of continuous production of natural gas wells, formation pressure and gas flow rate decrease continuously. The ability to carry liquid decreases continuously, thus gradually forming bottom hole liquid. Bottom hole liquid accumulation is an important reason for the decrease of production or shutdown of natural gas wells. How to diagnose whether there is liquid accumulation in natural gas wells and identify the degree of liquid accumulation, to adopt drainage gas recovery operation in time, is the research focus of efficient development of natural gas reservoirs. In this paper, a method for diagnosing bottom hole liquid accumulation combining production performance curve and modified Fernando inclined well critical liquid-carrying model is designed for a large scale double-branch horizontal well used in unconventional reservoirs. The method is applied to the Well X2 of He 8 Member in PCOC. The application results showed that there was no liquid accumulation in the horizontal and vertical sections of the Well X2. The liquid in the wellbore was generated at the bottom of the inclined section and the liquid accumulation is upward along the wellbore from the bottom of the inclined section, with the height of $3 \mathrm{~m}$.
\end{abstract}

\section{Introduction}

During natural gas production, condensate oil and water will be carried out of the formation. When the gas flow rate is lower than the critical flow rate of carrying liquid, these liquids will deposit at the bottom of the well and produce bottom hole liquid. Gas well liquid accumulation is a common problem in the middle and late stages of natural gas development. Once the formation of wellbore liquid accumulates, it will lead to a sharp decline in the production of gas wells and even lead to production cessation in serious cases. Therefore, the accurate calculation of liquid accumulation height is of great significance to the reasonable implementation of drainage gas recovery technology and the improvement of gas well production.

Turner et al. ${ }^{[1]}$ first put forward Turner's single drop model for the establishment of the gas well liquid accumulation model. The reason for liquid accumulation in the gas well is analyzed, and the liquid drop model and liquid film model are established. Coleman et al. ${ }^{[2]}$ modified the coefficients of the Turner model, but the Coleman model was only validated for low-pressure gas wells with wellhead pressures of less than 500psi. Li Min et al. ${ }^{[3]}$ established Li Min's model and assumed that the form of the liquid in the gas wellbore was an elliptical droplet. Belfroid et al. ${ }^{[4]}$ took into account the influence of the tilt angle of the string on the force of the droplet and deduced the calculation model of the critical liquid-carrying gas flow rate suitable for the inclined tube with the angle range $5^{\circ}$ to $90^{\circ}$. Zhou et al. considered that wellbore liquid holdup is also an important factor affecting the critical gas flow rate of liquid-carrying gas wells ${ }^{[5,6]}$. However, these critical liquid-carrying models are more complex to calculate the bottom hole liquid height, and few of them apply to the calculation of the bottom hole liquid height of doublebranch horizontal wells. Therefore, based on the field production data of $\mathrm{PCOC}$, this paper optimized the critical liquid-carrying model suitable for the doublebranch horizontal wells of the He 8 Member. The following aspects are studied: (1) Diagnose the liquid accumulation, determine whether there is the liquid accumulation in the wellbore, and determine the beginning time of liquid accumulation. (2) Establish a critical liquid-carrying model to identify the degree of liquid accumulation in the vertical, inclined and horizontal sections, and determine the height of liquid accumulation. (3) Take Well X2 of He 8 Member in PCOC as an example, the bottom hole liquid is diagnosed and the degree of liquid is identified.

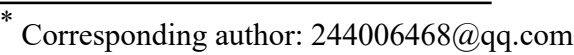




\section{Optimization of critical liquid-carrying model}

\subsection{Critical liquid-carrying model of the vertical well section}

At present, the Turner model, Coleman model, and Li Min model have been widely adopted in the vertical section. Based on the static pressure test results of the $\mathrm{He}$ 8 Member in PCOC, this paper selects the critical liquidcarrying model for the vertical section of gas wells with liquid accumulation. The comparison between the critical liquid-carrying flow rate of the three gas models and the actual gas flow rate at various parts of the wellbore is shown in Fig.1.

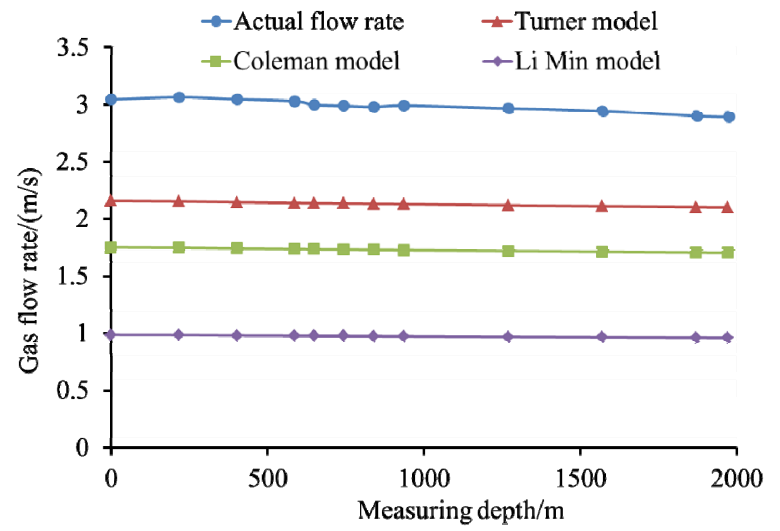

Fig. 1. Comparison of actual gas flow rate and critical liquidcarrying flow rate.

As shown in Fig.1, the gas flow rate in the vertical section is larger than the value calculated by the three critical gas carrying models, even after liquid accumulation has occurred in the wellbore. Therefore, the critical gas carrying flow rate model for doublebranch horizontal wells can not be determined only by the data of the vertical well section. It is necessary to determine the gas critical liquid-carrying flow rate model suitable for the double-branch horizontal well in PCOC through the relevant data of the inclined well section.

\subsection{Critical liquid-carrying model of the inclined well section}

Flores-Avila et al. ${ }^{[7,8]}$ studied the law of gas carrying liquid in double-branch horizontal wells. Based on the Turner model, the influence of inclination angle on the critical liquid-carrying flow rate is considered, and the judgment model of the critical liquid-carrying flow rate in the inclined section is deduced. The validity of the model is verified by experiments. The specific form of Fernando's model is shown in Eq 1.

$$
v_{\text {Scrit }}=4.86\left[\frac{\sigma\left(\rho_{L}-\rho_{g}\right)}{K_{d} \cos \alpha \rho_{g}{ }^{2}}\right]^{0.25}
$$

Where $v_{\text {scrit }}$ is the critical liquid-carrying flow rate of gas, $\mathrm{m} / \mathrm{s} ; \sigma$ is the gas-liquid interfacial tension $\mathrm{N} / \mathrm{m} ; \rho_{L}$ is the density of liquid, $\mathrm{kg} / \mathrm{m}^{3} ; \rho_{g}$ is the density of gas, $\mathrm{kg} / \mathrm{m}^{3} ; \alpha$ is the inclination angle; $K_{d}$ is the drag coefficient.

After repeated fitting and combining calculation parameters, when the drag coefficient $K_{d}$ is 15 , the modified Turner model proposed by Fernando considering the inclination angle has a good fit with the actual test data of the double-branch horizontal well of He 8 Member in PCOC. Therefore, the modified formula proposed by Fernando is selected for analysis in this paper and is used as the basis for judging the liquid accumulation problem of double-branch horizontal wells.

\section{A new method for diagnosis of bottom hole liquid accumulation in double- branch horizontal wells}

Aiming at the problems existing in the diagnosis of liquid accumulation height in double-branch horizontal wells, a liquid accumulation identification method combining the production decline curve and IPR curve is proposed. For the wells with liquid accumulation, this method can be used to monitor the position and height of the liquid accumulation page in real-time, which can provide a strong basis for taking reasonable and effective drainage gas recovery measures, to improve the development efficiency of the oilfield to a certain extent and delay the production decline degree of the gas well. The basic calculation steps are as follows:

(1) According to the gas well production test data, draw Arps production decline curve.

(2) Three production decline laws of exponential decline, hyperbolic decline, and harmonic decline were fitted to select the optimal production decline law and determine the decline rate.

(3) Combine the actual production curve of the well with the curve of the production decline law of the well in one graph, and compare the size of the two. When the actual production is always smaller than the output calculated by the production decline law, this point is the time when the liquid begins to accumulate.

(4) Draw the IPR curve of the well at a certain time, diagnose the liquid in the vertical section, inclined section, and horizontal section of the double-branch horizontal well, and calculate the critical liquid-carrying flow.

(5) Compare the actual gas flow rate and the critical liquid-carrying flow rate at different well sections, and determine the well depth when the actual flow rate is below the critical liquid-carrying flow rate, to calculate the liquid level position and the height of liquid accumulation.

\section{Case analysis}

(1) Draw Arps production decline curve.

Based on the production data of the Well X2 and the Arps production decline theory, the production data of the Well X2 were screened with a time interval of 60 
days. The corresponding relationship curve between production and time is shown in Fig.2.

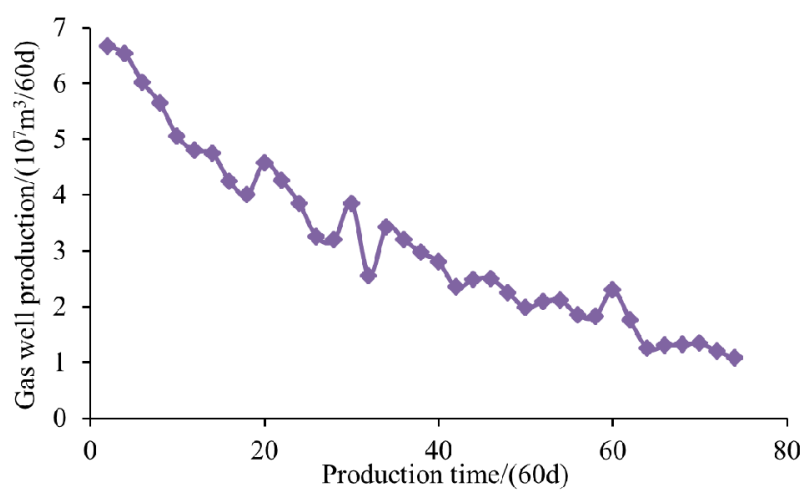

Fig. 2. The relationship between production time and production of Well X2.

(2) Three kinds of production decline laws are optimized to determine the decline rate.

The curve data points are fitted with an exponential decline, hyperbolic decline, and harmonic decline laws respectively. It can be concluded from the fitting results that the exponential decreasing fitting coefficient is the highest among the three fitting methods, and the decline rate is determined to be $0.0227 / 60$ days, and the converted daily production decline rate is $3.783 \times 10^{-4}$. The initial production of the well is determined to be $121.39 \times 10^{4} \mathrm{~m}^{3} / \mathrm{d}$. Eq. 2 is the relationship of the production decline law of Well X2.

$$
q=121.39 \times 10^{4} e^{-3.783 \times 10^{-4} t}
$$

Where $q$ is production, $\mathrm{m}^{3} / \mathrm{d} ; t$ is time, $\mathrm{d}$.

(3) Determine when the liquid begins to accumulate.

Combining the production decline law curve of Well $\mathrm{X} 2$ with the actual production data of the well, the two are combined in a single figure. The actual production of the well fluctuated up and down the production decline curve, but after April 2014 the actual production of the well was always lower than that of the production decline law. So the initial determination was that the well began to collect liquid in April 2014.

(4) Diagnosis of liquid accumulation based on IPR curve.

When calculating the productivity of this gas well, the Giger gas well productivity evaluation model is adopted. The specific form of the Giger productivity evaluation model ${ }^{[9]}$ is shown in Eq. 3.

$$
p_{e}{ }^{2}-p_{w f}{ }^{2}=a Q+b Q^{2}
$$

Where $p_{w f}$ is the bottom hole pressure of horizontal well, MPa; $p_{e}$ is the original formation pressure, MPa; $a$, $b$ are coefficients; $Q$ is gas-well production, $\mathrm{m}^{3} / \mathrm{d}$.

The Gray model is used as the calculation formula for wellbore pressure. Eq. 4 is the pressure drop gradient equation $^{[10]}$.

$$
\frac{d p}{d z}=\frac{f \rho_{n} V_{m}^{2}}{2 d}+\rho_{m} g-\rho_{n}{ }^{2} V_{m}{ }^{2} \frac{d}{d z}\left(\frac{1}{\rho_{n}}\right)
$$

Where $\rho_{m}$ is the density of the mixture, $\mathrm{kg} / \mathrm{m}^{3} ; f$ is friction resistance coefficient; $V_{m}$ is the apparent flow rate of the gas-liquid mixture, $\mathrm{m} / \mathrm{s} ; \rho_{n}$ is natural gas density, $\mathrm{kg} / \mathrm{m}^{3} ; d$ is the wellbore radius, $\mathrm{m} ; g$ is the acceleration of gravity, $\mathrm{m} / \mathrm{s}^{2}$.

Take the average production data of May 2016, put the well structure and physical property parameters of Well X2 into Eq. 3 to calculate the production of Well $\mathrm{X} 2$ and the respective production of the two branches. Draw the inflow and outflow performance curve of Well $\mathrm{X} 2$, as shown in Fig. 3 .

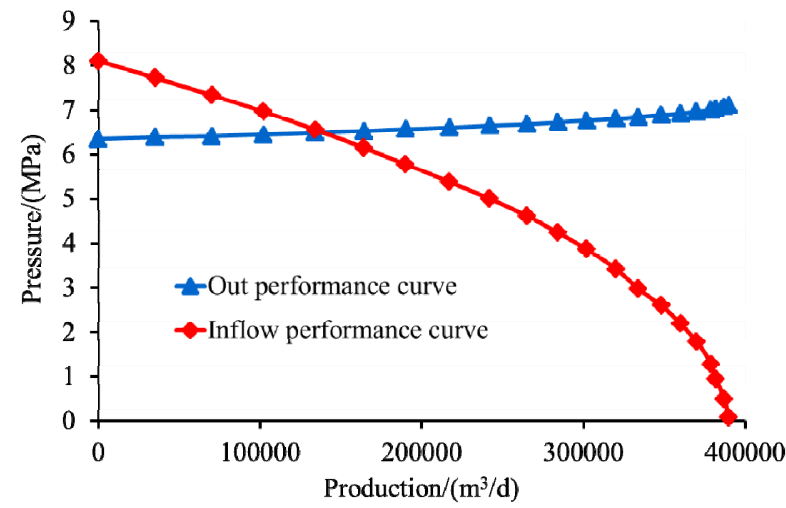

Fig. 3. Inflow and outflow performance curve of Well X2.

Fig.3 shows the calculated inflow and outflow performance curve of the Well X2 in May 2016. The average daily production of the Well X2 was $\mathrm{Q}=$ $137,289.4 \mathrm{~m}^{3} / \mathrm{d}$. Among them, the output of branch 1 is $67,126.2 \mathrm{~m}^{3} / \mathrm{d}$, and the output of branch 2 is $70,163.2 \mathrm{~m}^{3} / \mathrm{d}$. The actual flow rate and critical liquid-carrying flow rate of each part of the wellbore were calculated under the formation pressure and wellhead pressure.

The wellhead pressure is $4.89 \mathrm{MPa}$ and the temperature is $20^{\circ} \mathrm{C}$. After the well structure and the gas well physical property parameters are put into Eq. 4, the pressure distribution, temperature distribution, and gas flow rate variation in the wellbore are calculated.

First, the actual flow rate is compared with the critical liquid-carrying flow rate at each part of the vertical section of Well X2 under the formation pressure and wellhead pressure. The comparison results are shown in Table 1 below.

Table 1. Comparison of gas flow rate and critical liquidcarrying flow rate in the vertical section of Well X2.

\begin{tabular}{|c|c|c|}
\hline $\begin{array}{c}\text { Measuring } \\
\text { depth/(m) }\end{array}$ & $\begin{array}{c}\text { Gas flow } \\
\text { rate/(m/s) }\end{array}$ & $\begin{array}{c}\text { Critical liquid-carrying } \\
\text { flow rate/(m/s) }\end{array}$ \\
\hline 0 & 2.8445 & 1.1163 \\
\hline 405.4 & 2.8499 & 1.1104 \\
\hline 862.2 & 2.8209 & 1.1041 \\
\hline 1349 & 2.764 & 1.0977 \\
\hline
\end{tabular}

It can be seen from Table 1 that the actual gas flow rate at all parts of the vertical section of the Well X2 is 
higher than the critical liquid-carrying flow rate, so there will be no liquid accumulation in the vertical section.

Then, the actual flow rate is compared with the critical liquid-carrying flow rate of each part of the wellbore in the inclined section of the Well X2 under the formation pressure and wellhead pressure. The comparison results are shown in Table 2 below.

Table 2. Comparison of gas flow rate and critical liquidcarrying flow rate in the inclined section of Well X2.

\begin{tabular}{|c|c|c|}
\hline $\begin{array}{c}\text { Measuring } \\
\text { depth/(m) }\end{array}$ & $\begin{array}{c}\text { Gas flow } \\
\text { rate/(m/s) }\end{array}$ & $\begin{array}{c}\text { Critical liquid- } \\
\text { carrying flow } \\
\text { rate/(m/s) }\end{array}$ \\
\hline 1474.1 & 2.765 & 1.0874 \\
\hline 1857.4 & 2.7351 & 1.0902 \\
\hline 2306.2 & 2.7134 & 1.0990 \\
\hline 3278.9 & 2.6589 & 1.9638 \\
\hline 3375.7 & 0.5715 & 2.4617 \\
\hline 3401.3 & 0.5715 & 2.6208 \\
\hline
\end{tabular}

It can be seen from Table 2 that the actual gas flow rate at the bottom of the inclined section, the measuring depth $3375.7 \mathrm{~m}$ and $3401.3 \mathrm{~m}$, is less than the critical liquid-carrying flow rate, while the gas flow rate at the measuring depth of $3278.9 \mathrm{~m}$ is greater than the critical liquid-carrying flow rate. Therefore, there must be liquid accumulation at the bottom of the inclined section. The casing running depth was measured at $3401.3 \mathrm{~m}$, while the tubing running depth was measured at $3306.7 \mathrm{~m}$. And the casing's inner diameter is much larger than the tubing's inner diameter. Therefore, the gas flow rate in the casing outside the tubing at the bottom of the inclined section is small and less than the critical liquidcarrying flow rate and continues to produce liquid accumulation. After gas enters the tubing, the inner diameter decreases, and the flow rate increases, which is higher than the critical liquid-carrying flow rate. At this point, the gas increases its liquid-carrying capacity and pulls the liquid out of the tubing. So the liquid in the inclined section is generated in the casing outside the tubing at the bottom of the deviated section. The length of this part of the casing is the part of the borehole measuring depth of $3306.7-3401.3 \mathrm{~m}$, and the corresponding vertical depth is about $2847.86-2850.86 \mathrm{~m}$. The liquid accumulation site in the inclined section is at the vertical depth of $2847.86-2850.86 \mathrm{~m}$. The height of the liquid column is $3 \mathrm{~m}$.

According to the above calculation and analysis of the productivity of Well X2, the average monthly daily production of Well X2 in May 2016 was Q $=137289.4$ $\mathrm{m}^{3} / \mathrm{d}$, of which the production of branch 1 was $67126.2 \mathrm{~m}^{3} / \mathrm{d}$ and branch 2 was $70163.2 \mathrm{~m}^{3} / \mathrm{d}$. The inflow performance curves of the two branches of the doublebranch horizontal well are shown in Fig.4.

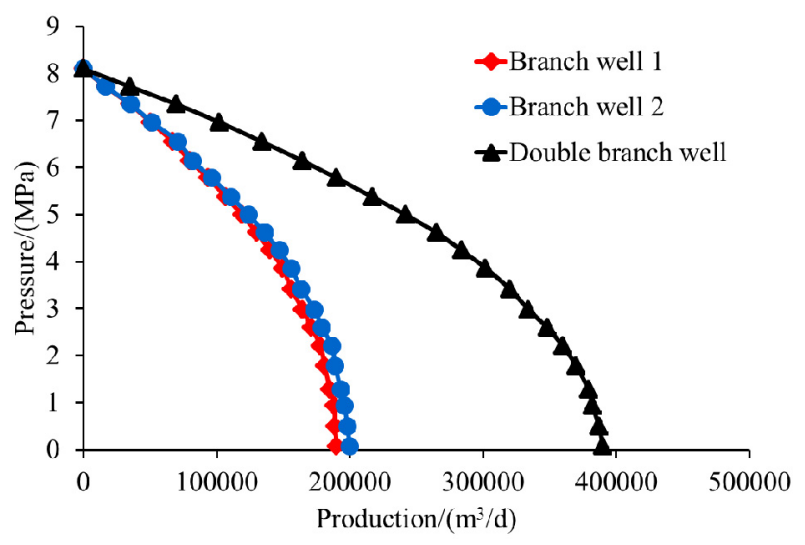

Fig. 4. The inflow performance curve of the two branches in Well X2.

The gas flow rate in two horizontal wells is converted by the production of double-branch horizontal wells and the gas volume coefficient. In May 2016, the formation pressure of Well X2 decreased to $8.1 \mathrm{MPa}$, bottom hole temperature was set at $89.2^{\circ} \mathrm{C}$, and $\mathrm{Z}$ value was 0.9 , then the compressibility coefficient of the gas in the horizontal wellbore in the reservoir could be calculated as $\mathrm{Bg}$ of 0.0139 . The average daily production of the two branches wells of the Well X2 was converted into daily production under formation pressure and temperature. Combined with the length and radius of the horizontal wellbore, the gas flow rate in the horizontal wellbore could be converted as follows: the gas flow rate in the horizontal well branch 1 was $\mathrm{v}_{1}=0.2953 \mathrm{~m} / \mathrm{s}$; the gas flow rate in horizontal well branch 2 was $\mathrm{v}_{2}=0.2825 \mathrm{~m} / \mathrm{s}$.

The liquid flow rate in double-branch horizontal wells is much lower than the gas flow rate. According to the Mandhane flow pattern division diagram of the horizontal wellbore ${ }^{[11]}$, it can be concluded that the flow pattern in two horizontal wells of Well X2 is stratified flow. This indicates that there is no liquid accumulation in the horizontal wellbore.

In summary, no liquid accumulation occurred in the horizontal and vertical sections of the Well X2. The liquid in the wellbore was generated at the bottom of the inclined section and proceeded up the wellbore from the bottom of the inclined section to a height of $3 \mathrm{~m}$.

\section{Conclusion}

In this paper, the Well X2 of the double-branch horizontal well of He 8 Member in PCOC is taken as an example for specific analysis, and the conclusions are as follows:

(1) For wells with liquid accumulation, the critical gas carrying flow rate model for double-branch horizontal wells can not be determined only by the data of the vertical well section. It is necessary to simulate the model by Fernando model of inclined well section.

(2) A method for diagnosis and identification of liquid accumulation in double-branch horizontal wells is proposed. The production decline law curve of gas well is drawn, the actual production curve is compared with the production decline law curve of the well, and the time point for starting liquid accumulation is determined. 
According to the IPR curve of the well at a certain time, the liquid accumulation diagnosis was carried out for the vertical section, inclined section and horizontal section of the double-branch horizontal well. The critical liquidcarrying flow rate was calculated to determine the position and height of the liquid accumulation.

(3) The application results of Well X2 in PCOC show that no liquid accumulation occurs in both horizontal and vertical sections. The liquid accumulation in the wellbore occurs at the bottom of the inclined section, and the liquid accumulation is upward along the wellbore from the bottom of the inclined section, with the height of $3 \mathrm{~m}$.

\section{Acknowledgments}

This work was supported by the National Natural Science Foundation of China (51874242). The authors would like to appreciate reviewers and editors whose critical comments were very helpful in preparing this article.

\section{References}

1. Turner R G, Hubbard M G, Dukler A E. Analysis and prediction of minimum flow rate for the continuous removal of liquids from gas wells[J]. Journal of Petroleum technology, 1969, 21(11): $1,475-1,482$.

2. Coleman S B, Clay H B, McCurdy D G, et al. A new look at predicting gas-well load-up[J]. Journal of Petroleum Technology, 1991, 43(03): 329-333.

3. Li M, Lei S, Li S. New view on continuous-removal liquids from gas wells[C]//SPE Permian basin oil and gas recovery conference. Society of Petroleum Engineers, 2001.

4. Belfroid S, Schiferli W, Alberts G, et al. Predicting onset and dynamic behaviour of liquid loading gas wells[C]//SPE Annual Technical Conference and Exhibition. Society of Petroleum Engineers, 2008.

5. Yuan H, Zhou D. Evaluation of two-phase flow correlations and Mechanistic models for pipelines at inclined downward flow[C]//SPE Eastern Regional/AAPG Eastern Section Joint Meeting. Society of Petroleum Engineers, 2008.

6. Zhou D, Yuan H. A new model for predicting gaswell liquid loading $[\mathrm{J}]$. SPE Production \& Operations, 2010, 25(02): 172-181.

7. Flores-Avila F S, Smith J R, Bourgoyne Jr A T, et al. Experimental evaluation of control fluid fallback during off-bottom well control: Effect of deviation angle $[\mathrm{C}] / / \mathrm{IADC} / \mathrm{SPE}$ Drilling Conference. Society of Petroleum Engineers, 2002.

8. Flores-Avila F S, Smith J R, Bourgoyne Jr A T. New dynamic kill procedure for off-bottom blowout wells considering counter-current flow of kill fluid[C]/SPE/IADC Middle East Drilling Technology Conference and Exhibition. Society of Petroleum Engineers, 2003.
9. Nakagawa E Y. Gas Kick Behavior During Well Control Operations in Vertical and Slanted Wells[J]. 1990.

10. Hasan A R, Kabir C S. Determining bottomhole pressures in pumping wells $[\mathrm{J}]$. Society of Petroleum Engineers Journal, 1985, 25(06): 823-838.

11. Standing M B, Katz D L. Density of natural gases[J]. Transactions of the AIME, 1942, 146(01): 140-149. 